\title{
ON WEIGHTED INEQUALITIES WITH GEOMETRIC MEAN OPERATOR
}

\section{DAH-CHIN LUOR}

\author{
(Received 28 February 2008)
}

\begin{abstract}
We give a characterization of pairs of weights for the validity of weighted inequalities involving certain generalized geometric mean operators generated by some Volterra integral operators, which include the Hardy averaging operator and the Riemann-Liouville integral operators. The estimations of the constants are also discussed. Our results generalize the work done by J. A. Cochran, C.-S. Lee, H. P. Heinig, B. Opic, P. Gurka, and L. Pick.
\end{abstract}

2000 Mathematics subject classification: primary 26D15; secondary 26D10.

Keywords and phrases: geometric mean operator, integral operator, weighted inequality, exponential inequality.

\section{Introduction}

Let $0<b \leq \infty, \Omega=\left\{(x, t) \in \mathbb{R}^{2} \mid 0<t<x<b\right\}$, and $\phi: \Omega \mapsto(0, \infty)$. Consider the Volterra integral operator

$$
T_{\phi} f(x):=\int_{0}^{x} \phi(x, t) f(t) d t, \quad f \geq 0,
$$

with $\phi$ satisfying the following conditions:

(Ф1) $\int_{0}^{x} \phi(x, t) d t=1$ for all $0<x<b$;

(Ф2) for any $r>0$, there exists $M(r)>0$ such that

$$
\exp \left(\int_{0}^{x} \phi(x, t) \log \left[\phi(x, t)^{-1} t^{r-1}\right] d t\right) \geq M(r) x^{r} \quad \forall 0<x<b .
$$

The geometric mean operator generated by $T_{\phi}$ is defined by

$$
G_{\phi} f(x):=\exp \left[T_{\phi} \log f(x)\right] .
$$

This research is supported by the I-Shou University of the Republic of China under grant ISU95-02-21.

(C) 2009 Australian Mathematical Society 0004-9727/09 \$A2.00+0.00 
In the case that $\phi$ is homogeneous of degree -1 , conditions ( $\Phi 1)$ and (Ф2) are satisfied if

$$
\int_{0}^{1} \phi(1, t) d t=1 \quad \text { and } \quad 0<\exp \left(\int_{0}^{1} \phi(1, t) \log \left[\phi(1, t)^{-1} t^{r-1}\right] d t\right)<\infty .
$$

In particular, they hold for $\phi(x, t)=\alpha t^{\alpha-1} / x^{\alpha}$ and $\phi(x, t)=\alpha(x-t)^{\alpha-1} / x^{\alpha}$, where $\alpha>0$.

This paper deals with the exponential inequality

$$
\left(\int_{0}^{b}\left(G_{\phi} f(x)\right)^{q} u(x) d x\right)^{1 / q} \leq C\left(\int_{0}^{b} f(x)^{p} v(x) d x\right)^{1 / p}
$$

where $0<p, q<\infty$, and $u, v$ are measurable functions defined on $(0, b)$, almost everywhere finite and positive. A considerable number of works are devoted to the study of (1.3). We refer the reader to Heinig et al. (see [7]), Opic and Gurka [22], Pick and Opic [26], and Persson and Stepanov [24] for $\phi(x, t)=1 / x$, Cochran and Lee [4], Čižmešija and Pečarić [3], Jain and Singh [12], and Jarrah and Singh [13] for $\phi(x, t)$ $=\alpha t^{\alpha-1} / x^{\alpha}$, Jain et al. [10] and Jain et al. [11] for $\phi(x, t)=h(t) / \int_{0}^{x} h(y) d y$, Heinig et al. [9], Heinig [8], and Love [15, 16] for $\phi$ to be homogeneous of degree -1 , Nassyrova et al. [20] and Persson et al. [25] for $\phi$ satisfying the Oinarov condition, and Kaijser et al. (see [14]) for general $\phi$.

The purpose of this paper is to extend the results in $[7,22,26]$ to more general $\phi$. Furthermore, we discuss some applications of our main result to the case that $\phi$ is homogeneous of degree -1 , including $\phi(x, t)=\alpha t^{\alpha-1} / x^{\alpha}$ and $\alpha(x-t)^{\alpha-1} / x^{\alpha}$ for $\alpha>0$. Our results are generalizations of works of $[4,7,8,9,22,26]$.

Throughout this paper we assume that all functions are measurable on their domains, and $u, v$ given in (1.3) are almost everywhere finite and positive. For $0<p<\infty$ and $\eta \geq 0$, define

$$
L_{p, \eta}^{+}:=\left\{f:(0, b) \mapsto[0, \infty] \mid \int_{0}^{b} f(x)^{p} \eta(x) d x<\infty\right\} .
$$

If $\eta \equiv 1$, we write $L_{p}^{+}$instead of $L_{p, \eta}^{+}$. For $0<z<\infty$, we define $z^{*}$ by $1 / z+1 / z^{*}=1$. We also take $\exp (-\infty)=0, \log 0=-\infty$, and $0 \cdot \infty=0$.

\section{Preliminaries}

To prove the main results, we need a key tool which is given by Muckenhoupt, Bradley, and Maz'ja (see [2, 18, 19, 23, 27, 29]) as follows.

Theorem 2.1. Let $0<p, q<\infty, p>1$, and $0<b \leq \infty$. Suppose that $\rho$ and $\eta$ are nonnegative functions, and $\eta^{1-p^{*}}$ is locally integrable. Then

$$
\left(\int_{0}^{b}\left(\int_{0}^{x} f(t) d t\right)^{q} \rho(x) d x\right)^{1 / q} \leq C\left(\int_{0}^{b} f(x)^{p} \eta(x) d x\right)^{1 / p}
$$


holds for all $f \in L_{p, \eta}^{+}$if and only if $A<\infty$, where

$$
A= \begin{cases}\sup _{0<\xi<b}\left(\int_{\xi}^{b} \rho(x) d x\right)^{1 / q}\left(\int_{0}^{\xi} \eta(x)^{1-p^{*}} d x\right)^{1 / p^{*}} & \text { if } p \leq q, \\ \left\{\int_{0}^{b}\left(\int_{x}^{b} \rho(t) d t\right)^{r / q}\left(\int_{0}^{x} \eta(t)^{1-p^{*}} d t\right)^{r / q^{*}} \eta(x)^{1-p^{*}} d x\right\}^{1 / r} & \text { if } q<p,\end{cases}
$$

and $1 / r=1 / q-1 / p$. Moreover, the best constant $C$ in (2.1) satisfies

$$
\begin{cases}A \leq C \leq\left(1+\frac{q}{p^{*}}\right)^{1 / q}\left(1+\frac{p^{*}}{q}\right)^{1 / p^{*}} A & \text { if } 1<p \leq q<\infty \\ q^{1 / q}\left(\frac{p^{*} q}{r}\right)^{1 / q^{*}} A \leq C \leq q^{1 / q}\left(p^{*}\right)^{1 / q^{*}} A & \text { if } 1 \leq q<p<\infty \\ q^{1 / q}\left(p^{*}\right)^{1 / q^{*}} A \leq C \leq q^{1 / q} A & \text { if } 0<q<1<p<\infty\end{cases}
$$

The following Lemma 2.2 deals with the existence of $G_{\phi} f(x)$ for $f \in L_{p, v}^{+}$.

LEMMA 2.2. Let $p>0$, $\phi$ satisfy $(\Phi 1)$, and $\int_{0}^{x} \phi(x, t) \log \phi(x, t) d t$ be finite for all $0<x<b$. Suppose that $v$ is almost everywhere finite and positive, and (2.4) holds:

$T_{\phi} \log (1 / v)(x)$ is well defined and $T_{\phi} \log (1 / v)(x)<\infty$ for all $0<x<b$.

Then, for all $f \in L_{p, v}^{+}, G_{\phi} f(x)$ exists and is finite for all $0<x<b$.

Proof OF LemMa 2.2 We first prove that if $h \in L_{1}^{+}$, then $G_{\phi} h(x)$ exists for all $0<x<b$. Suppose that $\int_{0}^{b} h(t) d t<\infty$. Then, for any $0<x<b$, $\int_{0}^{x} h(t) d t=\int_{0}^{x} \phi(x, t) \phi(x, t)^{-1} h(t) d t<\infty$. By [6, Theorem 187], $\int_{0}^{x} \phi(x, t)$ $\log \left[\phi(x, t)^{-1} h(t)\right] d t$ is well defined and

$\exp \left(\int_{0}^{x} \phi(x, t) \log \left[\phi(x, t)^{-1} h(t)\right] d t\right)=\lim _{r \rightarrow 0^{+}}\left\{\int_{0}^{x} \phi(x, t)\left(\phi(x, t)^{-1} h(t)\right)^{r} d t\right\}^{1 / r}$ exists and is finite. Since

$$
T_{\phi} \log h(x)=\int_{0}^{x} \phi(x, t) \log \phi(x, t) d t+\int_{0}^{x} \phi(x, t) \log \left[\phi(x, t)^{-1} h(t)\right] d t,
$$

we see that

$$
G_{\phi} h(x)=\exp \left(\int_{0}^{x} \phi(x, t) \log \phi(x, t) d t\right) \exp \left(\int_{0}^{x} \phi(x, t) \log \left[\phi(x, t)^{-1} h(t)\right] d t\right)
$$

exists and is finite for all $0<x<b$. For $f \in L_{p, v}^{+}$, let $h=f^{p} v$ and hence $h \in L_{1}^{+}$. Since $-\infty \leq T_{\phi} \log h(x)<\infty$,

$$
T_{\phi} \log f(x)=\frac{1}{p}\left(T_{\phi} \log h(x)+T_{\phi} \log (1 / v)(x)\right)
$$

and $G_{\phi} f(x)=G_{\phi} h(x)^{1 / p} G_{\phi}(1 / v)(x)^{1 / p}$ exists and is finite for all $0<x<b$. 
For any $s>0$, let $h^{s}=f^{p} v$. Then by a similar argument given in the proof of Lemma 2.2, we see that $G_{\phi} f(x)=G_{\phi} h(x)^{s / p} G_{\phi}(1 / v)(x)^{1 / p}$. This implies the following lemma.

Lemma 2.3. Let $0<p, q<\infty$ and $\phi$, $v$ be given in Lemma 2.2. Then (1.3) holds for all $f \in L_{p, v}^{+}$if and only if, for any $s>0$,

$$
\left(\int_{0}^{b}\left(G_{\phi} h(x)\right)^{s q / p} w(x) d x\right)^{p /(s q)} \leq C^{p / s}\left(\int_{0}^{b} h(x)^{s} d x\right)^{1 / s}
$$

holds for all $h \in L_{s}^{+}$with the same best constant $C$ as in (1.3). Here

$$
w(x)=G_{\phi}(1 / v)(x)^{q / p} u(x) .
$$

\section{Main results}

Let $0<p, q<\infty, \delta>1$, and $w$ be given by (2.6). We define

$$
A_{\delta}:= \begin{cases}\sup _{0<\xi<b} \xi^{(\delta-1) / p}\left(\int_{\xi}^{b} x^{-\delta q / p} w(x) d x\right)^{1 / q} & \text { if } p \leq q, \\ \left\{\int_{0}^{b}\left(\int_{x}^{b} t^{-\delta q / p} w(t) d t\right)^{p /(p-q)} x^{(\delta q-p) /(p-q)} d x\right\}^{(p-q) /(p q)} & \text { if } q<p .\end{cases}
$$

Our main result can be described as follows.

Theorem 3.1. Let $0<p, q<\infty$, $\phi$ satisfy ( $\Phi 1)$ to ( $\Phi 2)$, and (2.4) hold. Then (1.3) holds for all $f \in L_{p, v}^{+}$if and only if $A_{\delta}<\infty$ for all $\delta>1$. Moreover, the best constant $C$ in (1.3) satisfies

$$
\sup _{\delta>1} L_{\delta} A_{\delta} \leq C \leq \inf _{\delta>1} U_{\delta} A_{\delta}
$$

where

$$
U_{\delta}= \begin{cases}\inf _{s>1}\left(\frac{p+(s-1) q}{p}\right)^{1 / q}\left(\frac{p+(s-1) q}{(\delta-1) q}\right)^{(s-1) / p} M\left(\frac{\delta}{s}\right)^{-s / p} & \text { if } p \leq q, \\ \inf _{s>1}\left(\frac{s q}{p}\right)^{1 / q}\left(\frac{s-1}{\delta-1}\right)^{s / p-1 / q} \max \left\{\left(s^{*}\right)^{s / p-1 / q}, 1\right\} M\left(\frac{\delta}{s}\right)^{-s / p} & \text { if } q<p,\end{cases}
$$

and

$$
L_{\delta}= \begin{cases}\left(\frac{\delta-1}{\delta-1+\exp (-\varepsilon \delta)}\right)^{1 / p} & \text { if } p \leq q, \\ \left(\frac{\delta q-q}{p}\right)^{1 / q} \min \left(M_{l}^{(\delta q-p) /(p(p-q))}, M_{u}^{(\delta q-p) /(p(p-q))}\right) & \text { if } q<p .\end{cases}
$$


Here $\varepsilon \geq 0$ is the largest number that satisfies

$$
\int_{0}^{t} \phi(x, z) d z \geq \varepsilon t \phi(x, t) \quad \forall(x, t) \in \Omega
$$

and $M_{l}, M_{u}$ are positive constants that satisfy

$$
M_{l} x \leq \exp \left(\int_{0}^{x} \phi(x, t) \log t d t\right) \leq M_{u} x \quad \forall 0<x<b .
$$

If $\phi$ is homogeneous of degree -1 and $\int_{0}^{1} \phi(1, t) d t=1$, then

$$
\begin{aligned}
& \exp \left(\int_{0}^{x} \phi(x, t) \log \left[\phi(x, t)^{-1} t^{r-1}\right] d t\right) \\
& =x^{r} \exp \left(\int_{0}^{1} \phi(1, t) \log \left[\phi(1, t)^{-1} t^{r-1}\right] d t\right),
\end{aligned}
$$

and $(\Phi 2)$ is satisfied with $M(r)=\exp \left(\int_{0}^{1} \phi(1, t) \log \left[\phi(1, t)^{-1} t^{r-1}\right] d t\right)$ if this constant exists and is positive. Therefore, we now apply Theorem 3.1 to the case that $\phi$ is homogeneous of degree -1 and where it satisfies $(\Phi H 1)-(\Phi H 3)$ :

(ФH1) $\int_{0}^{1} \phi(1, t) d t=1$;

$(\Phi \mathrm{H} 2) \quad M_{1}=\exp \left(\int_{0}^{1} \phi(1, t) \log \phi(1, t) d t\right)<\infty$;

(ФH3) $M_{2}=\exp \left(\int_{0}^{1} \phi(1, t) \log t d t\right)>0$.

For such a case, $(\Phi 1)$ and $(\Phi 2)$ are satisfied. We may choose

$$
M(\delta / s)=\exp \left(\int_{0}^{x} \phi(x, t) \log \left[\phi(x, t)^{-1} t^{\delta / s-1}\right] d t\right) x^{-\delta / s}=M_{1}^{-1} M_{2}^{\delta / s-1}
$$

and $M_{l}=M_{u}=M_{2}$. The following theorem can be obtained from Theorem 3.1.

THEOREM 3.2. Let $0<p, q<\infty$, and let $\phi$ be homogeneous of degree -1 and satisfy (ФH1)-(ФH3). Suppose that (2.4) holds. Then (1.3) holds for all $f \in L_{p, v}^{+}$ if and only if $A_{\delta}<\infty$ for all $\delta>1$. The estimation of $C$ can be obtained by (3.2)-(3.4) with

$$
M(\delta / s)=M_{1}^{-1} M_{2}^{\delta / s-1}, \quad M_{l}=M_{u}=M_{2} .
$$

In the case when $p \leq q$,

$$
U_{\delta}= \begin{cases}M_{1}^{1 / p} M_{2}^{(1-\delta) / p} & \text { if } 1<\delta \leq \frac{M_{1} M_{2} p e}{q}+1, \\ \left(\frac{(\delta-1) q}{M_{1} M_{2} p}\right)^{1 / q} M_{1}^{1 / p} M_{2}^{(1-\delta) / p} e^{(1-\delta) /\left(M_{1} M_{2} p e\right)} & \text { if } \delta>\frac{M_{1} M_{2} p e}{q}+1,\end{cases}
$$

and hence $U_{\delta} \leq M_{1}^{1 / p} M_{2}^{(1-\delta) / p}$. 
In [9, Theorem 2.1] Heinig et al. gave a sufficient condition for (1.3) to hold if $b=\infty, \phi$ is homogeneous of degree -1 , and $\phi$ satisfies (ФH1) and (ФH3). On the other hand, Heinig [8] proved that in the case $p=q=1$, by adding some other conditions, the sufficient condition given in [9, Theorem 2.1] is also necessary. Our result yields a complete characterization of $u$ and $v$ in (1.3) for $\phi$ satisfying (ФH1)-(ФH3).

Consider the particular case $b=\infty, u(x)=x^{m}$, and $v(x)=x^{n}$. Then $w(x)$ defined by (2.6) reduces to $w(x)=M_{2}^{-n q / p} x^{m-(n q / p)}$. For $q<p, A_{\delta}=\infty$ for all $\delta>1$. If $p \leq q$ and $(m+1) / q=(n+1) / p$, then $A_{\delta}=M_{2}^{-n / p}(p /(\delta q-q))^{1 / q}$. Since $U_{\delta} \leq M_{1}^{1 / p} M_{2}^{(1-\delta) / p}$, by (3.2) and (3.3),

$$
C \leq M_{1}^{1 / p} M_{2}^{-n / p} \inf _{\delta>1} M_{2}^{(1-\delta) / p}\left(\frac{p}{\delta q-q}\right)^{1 / q}=M_{1}^{1 / p} M_{2}^{-n / p}\left(-e \log M_{2}\right)^{1 / q} .
$$

Therefore,

$$
\left(\int_{0}^{\infty}\left(G_{\phi} f(x)\right)^{q} x^{m} d x\right)^{1 / q} \leq M_{1}^{1 / p} M_{2}^{-n / p}\left(-e \log M_{2}\right)^{1 / q}\left(\int_{0}^{\infty} f(x)^{p} x^{n} d x\right)^{1 / p} .
$$

The following corollary considers the case when $\phi(x, t)=\alpha t^{\alpha-1} / x^{\alpha}$, where $\alpha>0$. For such a case,

$$
M_{1}=\alpha e^{1 / \alpha-1}, \quad M_{2}=e^{-1 / \alpha}, \quad \varepsilon=1 / \alpha .
$$

Corollary 3.3. Let $0<p, q<\infty$ and $\alpha>0$. Suppose that $u$, $v$ are almost everywhere finite and positive, and (2.4) holds with $\phi(x, t)=\alpha t^{\alpha-1} / x^{\alpha}$. Then

$$
\left(\int_{0}^{b}\left\{\exp \left(\frac{\alpha}{x^{\alpha}} \int_{0}^{x} t^{\alpha-1} \log f(t) d t\right)\right\}^{q} u(x) d x\right)^{1 / q} \leq C\left(\int_{0}^{b} f(x)^{p} v(x) d x\right)^{1 / p}
$$

holds for all $f \in L_{p, v}^{+}$if and only if $A_{\delta}<\infty$ for all $\delta>1$. The estimation of $C$ can be obtained by (3.2)-(3.4) with (3.5) and (3.8).

Consider the case $b=\infty$. For $\alpha=1$, Corollary 3.3 reduces to [22, Theorem] and [26, Corollary 3.10]. For general $\alpha$, inequality (3.9) was also investigated in $[9,12,13]$. In [9, Theorem 2.2], it was shown that (3.9) holds for all $f \in L_{p, v}^{+}$if and only if $A_{\alpha+1}<\infty$ (in the case when $p \leq q$ ) or $A_{p(\alpha+1) / q}<\infty$ (in the case when $q<p$ ). Corollary 3.3 contains these results; in addition, it also provides an estimation of $C$. If $p \leq q$ and $(m+1) / q=(n+1) / p$, then by (3.7),

$$
\begin{aligned}
& \left(\int_{0}^{\infty}\left\{\exp \left(\frac{\alpha}{x^{\alpha}} \int_{0}^{x} t^{\alpha-1} \log f(t) d t\right)\right\}^{q} x^{m} d x\right)^{1 / q} \\
& \leq \alpha^{1 / p-1 / q} \exp (1 / q+(n-\alpha+1) /(\alpha p))\left(\int_{0}^{\infty} f(x)^{p} x^{n} d x\right)^{1 / p}
\end{aligned}
$$


If $p=q=1$ and $m=n$, then (3.10) is the well-known Cochran-Lee's inequality.

We can also apply Theorem 3.2 to the case when $\phi(x, t)=\alpha(x-t)^{\alpha-1} / x^{\alpha}$, where $\alpha>0$. In this case,

$$
M_{1}=\alpha e^{1 / \alpha-1}, \quad M_{2}=e^{-\gamma-\Gamma^{\prime}(\alpha+1) / \Gamma(\alpha+1)}, \quad \varepsilon= \begin{cases}0 & \text { for } 0<\alpha<1 \\ 1 & \text { for } \alpha \geq 1\end{cases}
$$

The constant $M_{2}$ can be obtained by the following equalities:

$$
\log M_{2}=\alpha \int_{0}^{1} z^{\alpha-1} \log (1-z) d z=-\alpha \int_{0}^{1} \sum_{n=1}^{\infty} \frac{z^{n+\alpha-1}}{n} d z=-\gamma-\frac{\Gamma^{\prime}(\alpha+1)}{\Gamma(\alpha+1)}
$$

where $\gamma$ is the Euler constant and $\Gamma(x)$ is the gamma function. The last equality is based on [1, Theorem 1.2.5]. We have the following corollary.

Corollary 3.4. Let $0<p, q<\infty$ and $\alpha>0$. Suppose that $u$, $v$ are almost everywhere finite and positive, and (2.4) holds with $\phi(x, t)=\alpha(x-t)^{\alpha-1} / x^{\alpha}$. Then

$$
\begin{aligned}
& \left(\int_{0}^{b}\left\{\exp \left(\frac{\alpha}{x^{\alpha}} \int_{0}^{x}(x-t)^{\alpha-1} \log f(t) d t\right)\right\}^{q} u(x) d x\right)^{1 / q} \\
& \quad \leq C\left(\int_{0}^{b} f(x)^{p} v(x) d x\right)^{1 / p}
\end{aligned}
$$

holds for all $f \in L_{p, v}^{+}$if and only if $A_{\delta}<\infty$ for all $\delta>1$. The estimation of $C$ can be obtained by (3.2)-(3.4) with (3.5) and (3.11).

Another type of characterization can also be found in [20, Theorem 5.1] for the case when $0<p, q<\infty, \alpha>0$ and in [8, Corollary 3.1] for the case when $p=q=1$, $\alpha \geq 1$.

\section{Proof of Theorem 3.1}

We first prove the sufficient part. Suppose that $A_{\delta}<\infty$ for all $\delta>1$. Condition (Ф2) ensures that $\int_{0}^{x} \phi(x, t) \log \phi(x, t) d t$ is finite for all $0<x<b$. By Lemmas 2.2 and 2.3, inequality (1.3) holds for all $f \in L_{p, v}^{+}$if and only if (2.5) holds for all $h \in L_{s}^{+}$, where $s>1$. Since $\int_{0}^{x} \phi(x, t) \log \left[\phi(x, t)^{-1} t^{\delta / s-1} h(t)\right] d t$ is well defined, by Jensen's inequality and $(\Phi 2)$,

$$
\begin{aligned}
G_{\phi} h(x) & \leq \exp \left(-\int_{0}^{x} \phi(x, t) \log \left[\phi(x, t)^{-1} t^{\delta / s-1}\right] d t\right) \int_{0}^{x} t^{\delta / s-1} h(t) d t \\
& \leq M(\delta / s)^{-1} x^{-\delta / s} \int_{0}^{x} t^{\delta / s-1} h(t) d t .
\end{aligned}
$$


This implies that

$$
\begin{aligned}
& \int_{0}^{b}\left(G_{\phi} h(x)\right)^{s q / p} w(x) d x \\
& \quad \leq M(\delta / s)^{-s q / p} \int_{0}^{b}\left(\int_{0}^{x} t^{\delta / s-1} h(t) d t\right)^{s q / p} x^{-\delta q / p} w(x) d x .
\end{aligned}
$$

Replace $p, q, f(t), \rho(x)$, and $\eta(x)$ in Theorem 2.1 by $s, s q / p, t^{\delta / s-1} h(t)$, $x^{-\delta q / p} w(x)$, and $x^{s-\delta}$, respectively. Then (2.5) holds and

$$
C \leq \begin{cases}\left(\frac{p+(s-1) q}{p}\right)^{1 / q}\left(\frac{p+(s-1) q}{(\delta-1) q}\right)^{(s-1) / p} M(\delta / s)^{-s / p} A_{\delta} & \text { for } p \leq q, \\ \left(\frac{s q}{p}\right)^{1 / q}\left(\frac{s-1}{\delta-1}\right)^{s / p-1 / q} \max \left\{\left(s^{*}\right)^{s / p-1 / q}, 1\right\} M(\delta / s)^{-s / p} A_{\delta} & \text { for } q<p\end{cases}
$$

Since (4.2) is true for arbitrary $s>1$ and $\delta>1$, we have the upper estimations of $C$ given in (3.2) and (3.3).

In the following we prove the necessary part of Theorem 3.1. The idea is based on the proof of [24, Lemma 1] and [26, Lemma 3.2]. Consider the case $p \leq q$. By Lemma 2.3 , the inequality

$$
\left(\int_{0}^{b}\left(G_{\phi} h(x)\right)^{q / p} w(x) d x\right)^{1 / q} \leq C\left(\int_{0}^{b} h(x) d x\right)^{1 / p}
$$

holds for all $h \in L_{1}^{+}$with the same constant $C$ as in (1.3). Let $\xi>0, \delta>1$, and let $\varepsilon \geq 0$ be the largest number that satisfies $\int_{0}^{t} \phi(x, z) d z \geq \varepsilon t \phi(x, t)$ for all $(x, t) \in \Omega$. Let

$$
h(t)=\chi_{(0, \xi)}(t) \xi^{-1}+\chi_{(\xi, b)}(t) e^{-\varepsilon \delta} \xi^{\delta-1} t^{-\delta} .
$$

Then

$$
\left(\int_{0}^{b} h(x) d x\right)^{1 / p} \leq\left(\frac{\delta-1+e^{-\varepsilon \delta}}{\delta-1}\right)^{1 / p}
$$

On the other hand, for $\xi<x<b$,

$$
\begin{aligned}
\int_{0}^{x} \phi(x, t) \log h(t) d t= & \int_{0}^{\xi} \phi(x, t) \log \xi^{-1} d t+\int_{\xi}^{x} \phi(x, t) \log \left[e^{-\varepsilon \delta} \xi^{\delta-1} t^{-\delta}\right] d t \\
= & -\log \xi-\delta \int_{\xi}^{x} \phi(x, t) \log \left[\frac{t}{\xi}\right] d t \\
& -\varepsilon \delta \int_{\xi}^{x} \phi(x, t) d t
\end{aligned}
$$


Moreover, since

$$
\begin{aligned}
\int_{\xi}^{x} \phi(x, t) \log \left[\frac{t}{\xi}\right] d t & =\log \left[\frac{x}{\xi}\right] \int_{\xi}^{x} \phi(x, t) d t+\int_{\xi}^{x} \phi(x, t)\left(-\int_{t}^{x} \frac{1}{y} d y\right) d t \\
& =\log \left[\frac{x}{\xi}\right] \int_{\xi}^{x} \phi(x, t) d t-\int_{\xi}^{x} \frac{1}{y} \int_{\xi}^{y} \phi(x, t) d t d y \\
& =\log \left[\frac{x}{\xi}\right]-\int_{\xi}^{x} \frac{1}{y} \int_{0}^{y} \phi(x, t) d t d y
\end{aligned}
$$

we have

$$
\begin{aligned}
\int_{0}^{x} \phi(x, t) \log h(t) d t= & -\log \xi-\delta \log \left[\frac{x}{\xi}\right] \\
& +\delta \int_{\xi}^{x}\left(\frac{1}{t} \int_{0}^{t} \phi(x, z) d z\right)-\varepsilon \phi(x, t) d t \\
\geq & \log \left[\xi^{\delta-1} x^{-\delta}\right] .
\end{aligned}
$$

This shows that $G_{\phi} h(x) \geq \xi^{\delta-1} x^{-\delta}$ for $\xi<x<b$ and

$$
\int_{0}^{b}\left(G_{\phi} h(x)\right)^{q / p} w(x) d x \geq \xi^{(\delta-1) q / p} \int_{\xi}^{b} x^{-\delta q / p} w(x) d x .
$$

By (4.3)-(4.5),

$$
C\left(\frac{\delta-1+e^{-\varepsilon \delta}}{\delta-1}\right)^{1 / p} \geq \xi^{(\delta-1) / p}\left(\int_{\xi}^{b} x^{-\delta q / p} w(x) d x\right)^{1 / q} .
$$

Since (4.6) holds for all $0<\xi<b$,

$$
C \geq\left(\frac{\delta-1}{\delta-1+e^{-\varepsilon \delta}}\right)^{1 / p} A_{\delta}
$$

Inequality (4.7) is true for all $\delta>1$, and we have the lower estimation given in (3.2) and (3.4).

Consider the case $q<p$. Let $\left\{b_{n}\right\}$ be an increasing sequence which converges to $b$ and

$$
w_{n}(x)=[\min (w(x), n)] \chi_{\left(0, b_{n}\right)}(x)+\left[\min \left(w(x), x^{-2 q / r}\right)\right] \chi_{\left[b_{n}, b\right)}(x),
$$

where $1 / r=1 / q-1 / p$. For $\delta>1$, define

$$
h_{n}(x)=x^{(\delta q-p) /(p-q)}\left(\int_{x}^{b} t^{-\delta q / p} w_{n}(t) d t\right)^{p /(p-q)} \quad \text { for } 0<x<b .
$$


By the dual Hardy inequality (see [23, Theorem 6.2]), we see that

$$
\int_{0}^{b} h_{n}(x) d x \leq\left(\frac{p}{\delta q-q}\right)^{p /(p-q)} \int_{0}^{b} w_{n}(x)^{p /(p-q)} d x<\infty
$$

and $G_{\phi} h_{n}(x)$ exists and is finite for all $0<x<b$. Replace $h$ by $h_{n}$ in (4.3). Since $w_{n} \leq w$,

$$
\left(\int_{0}^{b}\left(G_{\phi} h_{n}(x)\right)^{q / p} w_{n}(x) d x\right)^{1 / q} \leq C\left(\int_{0}^{b} h_{n}(x) d x\right)^{1 / p} .
$$

By $(\Phi 2)$,

$$
\begin{aligned}
G_{\phi} h_{n}(x) & \geq\left\{\exp \left(\int_{0}^{x} \phi(x, t) \log \left[t^{(\delta q-p) /(p-q)}\right] d t\right)\right\}\left(\int_{x}^{b} t^{-\delta q / p} w_{n}(t) d t\right)^{p /(p-q)} \\
& \geq \tilde{M}^{p} x^{(\delta q-p) /(p-q)}\left(\int_{x}^{b} t^{-\delta q / p} w_{n}(t) d t\right)^{p /(p-q)}
\end{aligned}
$$

where $\tilde{M}=\min \left(M_{l}^{(\delta q-p) /(p(p-q))}, M_{u}^{(\delta q-p) /(p(p-q))}\right)$. Therefore,

$$
\begin{aligned}
& \int_{0}^{b}\left(G_{\phi} h_{n}(x)\right)^{q / p} w_{n}(x) d x \\
& \quad \geq \tilde{M}^{q} \int_{0}^{b}\left(\int_{x}^{b} t^{-\delta q / p} w_{n}(t) d t\right)^{q /(p-q)} x^{\left(\delta q^{2}-p q\right) /\left(p^{2}-p q\right)} w_{n}(x) d x \\
& \quad=\frac{(\delta q-q) \tilde{M}^{q}}{p} \int_{0}^{b}\left(\int_{x}^{b} t^{-\delta q / p} w_{n}(t) d t\right)^{p /(p-q)} x^{(\delta q-p) /(p-q)} d x
\end{aligned}
$$

By (4.8),

$$
C \geq\left(\frac{\delta q-q}{p}\right)^{1 / q} \tilde{M}\left\{\int_{0}^{b}\left(\int_{x}^{b} t^{-\delta q / p} w_{n}(t) d t\right)^{p /(p-q)} x^{(\delta q-p) /(p-q)} d x\right\}^{(p-q) /(p q)} .
$$

Let $n \rightarrow \infty$. Since $w_{n} \uparrow w$, we have $C \geq((\delta q-q) / p)^{1 / q} \tilde{M} A_{\delta}$. This holds for all $\delta>1$, so we have the lower estimation given in (3.2) and (3.4). This completes the proof.

\section{Concluding remarks}

REMARK 5.1. In [17, Theorem 2], Manakov showed that if $1<p<q<\infty, b=\infty$, and $\int_{0}^{\infty} \eta(x)^{1-p^{*}} d x=\infty$, then the upper estimation of $C$ given in (2.3) can be replaced by

$$
C \leq\left(\frac{\Gamma(q / \tau)}{\Gamma(1+1 / \tau) \Gamma((q-1) / \tau)}\right)^{\tau / q} A, \quad \text { for } \tau=q / p-1 .
$$


Hence if $0<p<q<\infty$ then $U_{\delta}$ given in (3.3) can be replaced by

$$
U_{\delta}=\inf _{s>1}\left(\frac{s-1}{\delta-1}\right)^{(s-1) / p}\left(\frac{\Gamma(s q /(q-p))}{\Gamma(q /(q-p)) \Gamma((s q-p) /(q-p))}\right)^{(q-p) /(p q)} M\left(\frac{\delta}{s}\right)^{-s / p} .
$$

REMARK 5.2. Suppose that $0<p, q<\infty, b=\infty, u$ and $v$ are positive and finite almost everywhere, $\phi$ satisfies $(\Phi 1)$ and ( $\Phi 2)$, and (2.4) holds. Define $w(x)=G_{\phi}(1 / v)$ $(x)^{q / p} u(x)$. By Theorem 3.1 and the results in [20, 24, 25, 28], the following (1)-(5) are equivalent.

(1)

$$
\left(\int_{0}^{\infty}\left(G_{\phi} f(x)\right)^{q} u(x) d x\right)^{1 / q} \leq C\left(\int_{0}^{\infty} f(x)^{p} v(x) d x\right)^{1 / p}, \quad f \in L_{p, v}^{+}
$$

(2) $A_{\delta}$ defined by (3.1) is finite for all $\delta>1$.

(3) For all $\delta>1$,

$$
\begin{aligned}
& \left(\int_{0}^{\infty}\left(\frac{1}{x} \int_{0}^{x} f(t) d t\right)^{\delta q / p} w(x) d x\right)^{p /(\delta q)} \\
& \leq C\left(\int_{0}^{\infty} f(x)^{\delta} d x\right)^{1 / \delta}, \quad f \in L_{\delta}^{+} .
\end{aligned}
$$

(4) For all $\delta>1$,

$$
\left(\int_{0}^{\infty} f(x)^{\delta q / p} w(x) d x\right)^{p /(\delta q)} \leq C\left(\int_{0}^{\infty} f(x)^{\delta} d x\right)^{1 / \delta}, \quad f \in L_{\delta}^{+} \text {and } f \downarrow
$$

where $f \downarrow$ means $f$ is nonincreasing.

(5) The constant $B$ is finite, where $B$ is defined by (5.6):

$$
B:= \begin{cases}\sup _{\xi>0} \xi^{-1 / p}\left(\int_{0}^{\xi} w(x) d x\right)^{1 / q} & \text { if } p \leq q, \\ \left\{\int_{0}^{\infty}\left(\frac{1}{x} \int_{0}^{x} w(t) d t\right)^{p /(p-q)} d x\right\}^{(p-q) /(p q)} & \text { if } q<p .\end{cases}
$$

The constant $C$ that occurs in (5.3)-(5.5) may be different. More equivalent conditions can also be found in $[5,21]$, but we leave the details to the reader.

\section{References}

[1] G. E. Andrews, R. Askey and R. Roy, Special Functions, Encyclopedia of Mathematics and its Applications, 71 (Cambridge University Press, Cambridge, 1999).

[2] J. S. Bradley, 'Hardy inequalities with mixed norms', Canad. Math. Bull. 21 (1978), 405-408. 
[3] A. Čižmešija and J. Pečarić, 'Some new generalisations of inequalities of Hardy and LevinCochran-Lee', Bull. Austral. Math. Soc. 63 (2001), 105-113.

[4] J. A. Cochran and C.-S. Lee, 'Inequalities related to Hardy's and Heinig's', Math. Proc. Cambridge Philos. Soc. 96 (1984), 1-7.

[5] A. Gogatishvili, A. Kufner, L.-E. Persson and A. Wedestig, 'An equivalence theorem for integral conditions related to Hardy's inequality', Real Anal. Exchange 2 (2004), 1-14.

[6] G. H. Hardy, J. E. Littlewood and G. Pólya, Inequalities, 2nd edn (Cambridge University Press, Cambridge, 1952).

[7] H. P. Heinig, 'Weighted inequalities in Fourier analysis', in: Nonlinear Analysis, Function Spaces and Applications, Vol. 4, Teubner-Texte Math., 119 (Teubner, Leipzig, 1990), pp. 42-85.

[8] _ - 'Exponential inequalities for a class of operators', Int. J. Math. Math. Sci. 31 (2002), 283-290.

[9] H. P. Heinig, R. Kerman and M. Krbec, 'Weighted exponential inequalities', Georgian Math. J. 8 (2001), 69-86.

[10] P. Jain, L.-E. Persson and A. P. Singh, 'On geometric mean inequalities with exponential weights', Soochow J. Math. 30 (2004), 391-400.

[11] P. Jain, L.-E. Persson and A. Wedestig, 'Carleman-Knopp type inequalities via Hardy inequalities', Math. Inequal. Appl. 4 (2001), 343-355.

[12] P. Jain and A. P. Singh, 'A characterization for the boundedness of geometric mean operator', Appl. Math. Lett. 13 (2000), 63-67.

[13] A. M. Jarrah and A. P. Singh, 'A limiting case of Hardy's inequality', Indian J. Math. 43 (2001), $21-36$.

[14] S. Kaijser, L. Nikolova, L.-E. Persson and A. Wedestig, 'Hardy-type inequalities via convexity', Math. Inequal. Appl. 8 (2005), 403-417.

[15] E. R. Love, 'Inequalities related to Knopp's inequality', J. Math. Anal. Appl. 137 (1989), 173-180.

[16] _ 'Inequalities related to those of Hardy and of Cochran and Lee', Math. Proc. Cambridge Philos. Soc. 99 (1986), 395-408.

[17] V. M. Manakov, 'On the best constant in weighted inequalities for Riemann-Liouville integrals', Bull. London Math. Soc. 24 (1992), 442-448.

[18] V. G. Maz'ja, Sobolev Spaces (Springer, Berlin, 1985).

[19] B. Muckenhoupt, 'Hardy's inequality with weights', Studia Math. 44 (1972), 31-38.

[20] M. Nassyrova, L.-E. Persson and V. D. Stepanov, 'On weighted inequalities with geometric mean operator generated by the Hardy-type integral transform', J. Inequal. Pure Appl. Math. 3 (2002), 48.

[21] C. A. Okpoti, L.-E. Persson and G. Sinnamon, 'An equivalence theorem for some integral conditions with general measures related to Hardy's inequality', J. Math. Anal. Appl. 326 (2007), 398-413.

[22] B. Opic and P. Gurka, 'Weighted inequalities for geometric means', Proc. Amer. Math. Soc. 120 (1994), 771-779.

[23] B. Opic and A. Kufner, Hardy-type Inequalities, Pitman Research Notes in Mathematics Series, 219 (Longman Scientific \& Technical, Harlow, 1990).

[24] L.-E. Persson and V. D. Stepanov, 'Weighted integral inequalities with the geometric mean operator', J. Inequal. Appl. 7 (2002), 727-746.

[25] L.-E. Persson, V. D. Stepanov and E. P. Ushakova, 'Equivalence of Hardy-type inequalities with general measures on the cones of non-negative respective non-increasing functions', Proc. Amer. Math. Soc. 134 (2006), 2363-2372.

[26] L. Pick and B. Opic, 'On the geometric mean operator', J. Math. Anal. Appl. 183 (1994), 652-662.

[27] G. J. Sinnamon, 'Weighted Hardy and Opial-type inequalities', J. Math. Anal. Appl. 160 (1991), 434-445. 
[28] G. Sinnamon, 'Hardy's inequality and monotonicity', in: Function Spaces, Differential Operators and Nonlinear Analysis (Mathematical Institute of the Academy of Sciences of the Czech Republic, Prague, 2005), pp. 292-310.

[29] G. Sinnamon and V. D. Stepanov, 'The weighted Hardy inequality: new proofs and the case $p=1$ ', J. London Math. Soc. 54 (1996), 89-101.

DAH-CHIN LUOR, Department of Applied Mathematics, I-Shou University, Ta-Hsu, Kaohsiung 84008, Taiwan

e-mail: dclour@isu.edu.tw 